\title{
The preventive effect of dexmedetomidine on paroxysmal sympathetic hyperactivity in severe traumatic brain injury patients who have undergone surgery: a retrospective study
}

\author{
Qilin Tang ${ }^{1,2}$, Xiang Wu ${ }^{1,2}$, Weiji Weng ${ }^{1,2}$, Hongpeng Li ${ }^{3}$, Junfeng Feng ${ }^{1,2}$, Qing Mao ${ }^{1,2}$, Guoyi Gao ${ }^{\text {Corresp.. }}{ }^{1,}$ \\ 2, Jiyao Jiang ${ }^{1,2}$ \\ ${ }^{1}$ Department of Neurosurgery, Ren Ji Hospital, School of Medicine, Shanghai Jiao Tong University, Shanghai, China \\ 2 Shanghai Institute of Head Trauma, Shanghai, China \\ 3 Department of Neurosurgery, Rizhao City Hospital of Traditional Chinese Medicine, Rizhao, Shandong Province, China \\ Corresponding Author: Guoyi Gao \\ Email address: gao3@sina.com
}

Background. Paroxysmal sympathetic hyperactivity (PSH) results and aggravates in secondary brain injury, which seriously affects the prognosis of severe traumatic brain injury patients. Although several studies have focused on the treatment of PSH, few have concentrated on its prevention.

Methods. Ninety post-operation (post-op) severe traumatic brain injury (sTBI) patients admitted from October 2014 to April 2016 were chosen to participate in this study. Fifty of the post-op sTBI patients were sedated with dexmedetomidine and were referred as the "dexmedetomidine group" (admitted from May 2015 to April 2016). The other 40 patients (admitted from October 2014 to May 2015) received other sedations and were referred as the "control group." The two groups were then compared based on their PSH scores and the scores and ratios of those patients who met the criteria of "probable", "possible" and "unlikely" using the PSH assessment measure (PSH-AM) designed by Baguley et al. (2014). The durations of the neurosurgery intensive care unit (NICU) and hospital stays and the Glasgow outcome scale (GOS) values for the two groups were also compared to evaluate the therapeutic effects and the patients' prognosis.

Results. The overall PSH score for the dexmedetomidine group was 5.26 \pm 4.66 , compared with $8.58 \pm 8.09$ for the control group. The difference between the two groups' PSH scores was significant $(P=$ $0.017)$. The score of the patients who met the criterion of "probable" was $18.33 \pm 1.53$ in the dexmedetomidine group and $22.63 \pm 2.97$ in the control group, and the difference was statistically significant $(P=0.045)$. The ratio of patients who were classified as "unlikely" between the two groups was statistically significant $(P=0.028)$; that is, $42(84 \%)$ in the dexmedetomidine group and $25(62.5 \%)$ in the control group. The differences in NICU, hospital stays and GOS values between the two groups were not significant.

Conclusion. Dexmedetomidine has a preventive effect on PSH in STBI patients who have undergone surgery. 
1 The preventive effect of dexmedetomidine on paroxysmal sympathetic

2 hyperactivity in severe traumatic brain injury patients who have undergone

3 surgery: a retrospective study

4 Qilin Tang ${ }^{1,2}$, Xiang Wu ${ }^{1,2}$, Weiji Weng ${ }^{1,2}$, Hongpeng Li $^{3}$, Junfeng Feng ${ }^{1,2}$, Qing Mao ${ }^{1,2}$, Guoyi Gao ${ }^{1,2}$, Jiyao Jiang ${ }^{1,2}$

5 Affiliations:

6 1. Department of Neurosurgery, Ren Ji Hospital, School of Medicine, Shanghai Jiao Tong University, Shanghai,

7 China

8 2. Shanghai Institute of Head Trauma, Shanghai, China

9 3. Department of Neurosurgery, Rizhao City Hospital of Traditional Chinese Medicine, Rizhao, Shandong Province,

10 China.

12 Corresponding Author:

13 Guoyi Gao ${ }^{1,2}$

14 Email address: gao3@sina.com 


\section{Abstract}

Background. Paroxysmal sympathetic hyperactivity (PSH) results and aggravates in secondary brain injury, which

seriously affects the prognosis of severe traumatic brain injury patients. Although several studies have focused on

the treatment of PSH, few have concentrated on its prevention.

19 Methods. Ninety post-operation (post-op) severe traumatic brain injury (sTBI) patients admitted from October 2014

to April 2016 were chosen to participate in this study. Fifty of the post-op sTBI patients were sedated with

Results. The overall PSH score for the dexmedetomidine group was 5.26 \pm 4.66 , compared with $8.58 \pm 8.09$ for the 
patients who met the criterion of "probable" was $18.33 \pm 1.53$ in the dexmedetomidine group and $22.63 \pm 2.97$ in the

31 values between the two groups were not significant.

Conclusion. Dexmedetomidine has a preventive effect on PSH in sTBI patients who have undergone surgery. control group, and the difference was statistically significant $(\mathrm{P}=0.045)$. The ratio of patients who were classified as "unlikely" between the two groups was statistically significant $(\mathrm{P}=0.028)$; that is, $42(84 \%)$ in the dexmedetomidine group and $25(62.5 \%)$ in the control group. The differences in NICU, hospital stays and GOS

\section{Introduction}

41 hyperthermia, diaphoresis, tachycardia, hypertension, tachypnea, and dystonic posturing (Baguley et al. 2014). Since

42 Wilder Penfield's first report on the syndrome, numerous terms have been used to describe it, including diencephalic 
45 suffer from this disorder (Baguley et al. 2007b; Dolce et al. 2008; Fearnside et al. 1993; Fernandez-Ortega et al.

46 2012; Fernandez-Ortega et al. 2006; Hendricks et al. 2007; Lv et al. 2010; Lv et al. 2011; Perkes et al. 2010; Perkes

47 et al. 2011; Rabinstein 2007).

2004). Unstable conditions such as high blood pressure or fever, can result in and aggravate secondary brain injury,

which is considered to be one of the main causes of unfavorable prognosis (Baguley 2008a; Baguley et al. 2007a;

52 hypermetabolic state, unopposed inflammation, and weight loss - all of which can lead to worse outcomes and

prolonged hospital stays (Choi et al. 2013; Mehta et al. 2008; Tracey 2007). Several studies have focused on how to

treat PSH, but few have concentrated on its prevention.

Dexmedetomidine is a new selective alpha-2 agonist that has been shown to decrease sympathetic activation, 
60 this article, we report that dexmedetomidine may have a preventive effect on PSH in postoperative patients who

61 have suffered severe traumatic brain injury.

\section{Methods}

\section{Study population}

Neurosurgery at Ren Ji Hospital from October 2014 to April 2016. Consecutive patients were included if they were

older than 18, had suffered sTBI, and had undergone an operation. Patients were excluded if they met any of the

following criteria: serious hepatic dysfunction (Child-Pugh class B or C); serious renal dysfunction (undergoing

dialysis before surgery; or serum creatinine $>445 \mu \mathrm{mol} / \mathrm{L}$ and/or blood urea nitrogen $>20 \mathrm{mmol} / \mathrm{L}$ in preoperative

laboratory examination); unstable haemodynamics at NICU admission, such as bradycardia and hypotension; death

occurring within $24 \mathrm{~h}$ after surgery. Of the 103 consecutively recorded patients, 13 were excluded based on the 


\section{Treatment protocol}

For the dexmedetomidine group, when each patient was placed in the NICU after his or her operation,

continuous infusion at $0.25 \sim 0.75 \mu \mathrm{g} /(\mathrm{kg} \cdot \mathrm{h})^{-1}$. For the control group, propofol or midazolam was administered by intravenous pump infusion. Propofol was initiated at $2 \mathrm{mg} /(\mathrm{kg} \cdot \mathrm{h})^{-1}$ and midazolam was initiated at $0.1 \mathrm{mg} /(\mathrm{kg} \cdot$

Verbal informed consent was obtained from patients' decision makers. 
abnormal signs and symptoms of PSH were observed by trained nurses and events were reported to the doctors in

features (the Clinical Feature Scale [CFS]). The numerical output of these two components are added together to

\section{Statistical analysis}


103

104

105

106

107

108

109

110

111

112 The demographic patient data are listed in Table 1.

113

114

115 mg. Dexmedetomidine was not administrated to the patients in the control group. The PSH-AM score of the patients variables. For the continuous variables, group comparisons were made using parametric t-tests if the data followed normal distribution. Otherwise, non-parametric Mann-Whitney-U tests were used. For the categorical variables, such as gender, diagnosis and surgery type, group comparisons were made using a chi-quadrat test or a Fisher's exact test if at least one value was $<5$. Significance was set at $p<0.05$. To assess the power of our study, we conducted power calculations by using $\mathrm{G}^{*}$ power (Faul et al. 2007).

\section{Results}

The data entry was completed in July 2016, when all of the patients had received their three-month follow-up.

The patients' data were collected for retrospective analysis. No significant differences were noted between the two groups in relation to age, gender, pre-operative GCS, diagnosis, time from injury to surgery, and type of surgery.

The patients in the dexmedetomidine group were sedated for a period of $5.46 \pm 2.82$ days, compared with $6.08 \pm 2.95$ in the control group $(\mathrm{p}=0.317)$. The total dose of dexmedetomidine given to each person was $4.79 \pm 2.47$ 
118

119

120

No significant differences were noted between the two groups regarding the ratio of patients who met the criteria of "probable" (three $(6 \%)$ in the dexmedetomidine group vs. eight $(12.5 \%)$ in the control group, $\mathrm{P}=0.056)$ and "unlikely" (3.67 \pm 2.40 in the dexmedetomidine group vs. $3.28 \pm 2.30$ in the control group, $\mathrm{P}=0.519)$. However, the score of patients who met the "probable" criterion in the dexmedetomidine group was $18.33 \pm 1.53$, compared with $22.63 \pm 2.97$ in the control group $(\mathrm{P}=0.045)$. Moreover, $42(84 \%)$ of the patients in the dexmedetomidine group met the "unlikely" criterion, compared with $25(62.5 \%)$ patients in the control group $(\mathrm{P}=0.028)$. No significant differences in the groups were established for patients who met the "possible" criterion, in number or score (Figures 1 and 2).

In NICU, the two groups had the same mortality of $20 \%$ (10 of 50 in the dexmedetomidine group and 8 of 40 in the control group). No more patients died after transfered to ordinary wards. The patients in the dexmedetomidine group stayed in the NICU for an average duration of $15.70 \pm 13.07$ days and in the hospital for $23.50 \pm 16.58$ days, compared with $20.65 \pm 16.74$ and $28.53 \pm 20.28$ days, respectively, in the control group $(\mathrm{P}=0.119$ and $\mathrm{P}=0.174)$ (Figure 3). The GOS value at discharge was $3.00 \pm 1.28$ in the dexmedetomidine group, compared with $2.75 \pm 1.15$ in the control group $(\mathrm{P}=0.338)$. The GOS vaule three months after sTBI in the dexmedetomidine group was $3.42 \pm 1.47$, compared with $3.05 \pm 1.43$ in the control group $(\mathrm{P}=0.234)$ (Figure 4, Table 1). The statistical power for the outcome 
133 measures described above is relatively low $(21 \%-42 \%)$.
134

Severe traumatic brain injury, which is associated with considerable mortality and morbidity, represents a significant public health problem around the world. PSH after sTBI is one of the important factors contributing to adverse outcomes. The first diagnostic criteria for PSH were published in 1993 (Fearnside et al. 1993). Since that time, different criteria have been proposed mainly due to the presence of the following signs and symptoms in the absence of other potential causes: fever, tachycardia, hypertension, tachypnea, excessive diaphoresis and extensor posturing, or severe dystonia (Baguley et al. 2014; Blackman et al. 2004; Dolce et al. 2008; Perkes et al. 2011; Rabinstein 2007). A PSH diagnosis is one of exclusion. Considering differential diagnoses such as sepsis, systemic inflammatory response syndrom, or sedation withdrawal is crucial, but sometimes difficult. Baguley et al. (2014) proposed a consensus of diagnostic criteria for $\mathrm{PSH}$ — specifically, a probabilistic system that assigned a diagnostic likelihood rather than providing a definitive diagnosis (Baguley et al. 2014). This diagnostic system enables medical workers to diagnose PSH more precisely and provides a quantization foundation for PSH evaluation.

The pathophysiology of PSH is unclear, and related theories have not been empirically tested. Initially, the cause of the condition was thought to be diencephalic discharges (Bhigjee et al. 1985; Penfield 1929). However, 
148 later studies identified no seizure activities in PSH using electroencephalography (Baguley et al. 2006; Boeve et al.

149

150

151

152

153

154

155

156

157

158

159

1998). The current consensus is that epilepsy is not the cause of PSH (Bullard 1987; Pranzatelli et al. 1991; Thorley

et al. 2001). The accepted model is the excitatory-inhibitory ratio (EIR) model (Baguley 2008b), which proposes

that the afferent stimulus is normally controlled by tonic inhibitory drive from diencephalic centers. Once the tonic

inhibition cycle is broken, there is a positive-feedback loop that produces sympathetic over-activity following any

afferent stimuli (Baguley et al. 2009a; Baguley et al. 2009b). This model explains how a normally non-noxious

stimulus can cause an uncontrolled sympathetic response.

For PSH, timely diagnosis, swift episode control, and reduced onset frequency are all crucial in improving

prognosis. Regarding the treatment of PSH, beta-blockers, which attenuate sympathetic activation, are now widely

used to control its onset (Do et al. 2000; Rabinstein \& Benarroch 2008; Sneed 1995). Morphine, a potent $\mu$-opioid

receptor agonist; Bromocriptine, a dopamine receptor agonist; and baclofen, a GABA receptor agonist, have also

been reported to successfully alleviate PSH episodes (Becker et al. 2000; Cuny et al. 2001; Ko et al. 2010; Russo \&

O'Flaherty 2000). However, as few cohort studies guiding PSH treatment exist and the underlying pathophysiology

remains unclear, treatment strategies frequently focus on controlling symptoms. For instance, antipyretics are

administered to treat hyperthermia, sedatives for agitation, and antihypertensive medications for hypertension (Choi 
163

164

165

et al. 2013).

release and enhance sympathetic inhibition to maintain the balance of the unregulated sympathetic feedback loop.

Two cases of dexmedetomidine being used to diminish PSH symptoms have been reported (Goddeau et al. 2007;

Kern et al. 2016), which may support the disconnection theory. Unlike a traditional sedative such as propofol, which acts on GABA receptors in the cortex, dexmedetomidine displays the actions of analgesia, sedation, and anxiety treatment by acting on the $\alpha 2$ adrenergic receptors in the locus coeruleus (LC) (Nelson et al. 2003). Patients sedated using dexmedetomidine can be awakened at any time to judge changes in their conscious state. It has also been reported that dexmedetomidine has a neuroprotective effect because it inhibits the apoptosis of nerve cells, protecting against local ischemia and slowing the progression of infarction (Cai et al. 2014; Cosar et al. 2009; Dahmani et al. 2005). These features make dexmedetomidine a commonly prescribed sedative in NICUs.

PSH prevention to decrease the number and severity of episodes has more significance in improving the outcome of sTBI, compared with the elimination of PSH episodes. Although several studies have focused on treating PSH, few have addressed its prevention. Thus, based on the routine use of dexmedetomidine for post-op sedation and data collected from patients, this study explored whether dexmedetomidine, which is reported to 
178

successfully eliminate PSH episodes, also has a preventive effect on this syndrome.

We compared the overall PSH-AM scores between the two groups and found that the score for the dexmedetomidine group was significantly lower than that for the control group, indicating that the patients in the dexmedetomidine group had a lower probability of PSH diagnosis. Compared with the control group, the dexmedetomidine group had a lower score of patients meeting the "probable" criterion and a larger ratio of patients meeting the "unlikely" criterion, with a statistically significant difference. The preceding results showed that dexmedetomidine, to some extent, prevented the patients in the dexmedetomidine group from the onset of PSH.

The differences in duration of the NICU and hospital stays and in the GOS values at three-month follow-up between the two groups did not reach statistical significance. sTBI is a heterogeneous disease and patients' post-op conditions are variable and protean. Many factors may contribute to an sTBI prognosis. Moreover, the statistical power for the above three outcome measures is relatively low $(21 \%-42 \%)$, which may be due to the small sample size. The low statistical power indicates low ability to distinguish the effect from random chance. This may be why our study did not reach a statistically significant difference for these three outcome measures.

The onset of PSH is often sudden and short in duration. In addition, the symptoms are often atypical and changeful, which can make a timely diagnosis difficult. Delayed diagnoses and treatment difficulties can result in 
193

194

195

196

198

prolonged NICU and hospital stays, higher medical costs, and poorer outcomes (Hinson \& Sheth 2012; Lv et al.

2011). Dexmedetomidine is a commonly used sedative in the NICU, and had a preventive effect on post-op PSH in patients with sTBI who have undergone surgery in our study, although the exact mechanism remains unclear. This feature makes it a promising medication for the sedation of post-op sTBI patients and for preventing PSH onset, both of which are significant in reducing postoperative complications, cutting down the duration of hospitalization, and improving prognosis.

This study had several limitations. First, it was a single-center study, and thus the findings may not be generalizable to other centers. The two groups were studied over different periods, during which there might have been differences aside from the sedative used after surgery which were failed to recognize. Second, only those sTBI patients who underwent surgery were studied, and therefore these findings may not be generalizable to those who have not undergone surgery. Third, we observed only PSH episodes during hospital stays without long-term followup after discharge. Fourth, limited by objective conditions, we evaluated GOS after three months based on the descriptions provided by the patients' relatives or health care givers, which might cause bias. And last, the statistical power for this study is relatively low (21\%-42\%), which indicates low ability to distinguish the differences. The preceding limitations impair, to some extent, the trustworthiness of our conclusions. 
208 Conclusions

\section{Acknowledgements}

\section{Reference}

Baguley IJ. 2008a. Autonomic complications following central nervous system injury. Semin Neurol 28:716-725. 10.1055/s-0028-1105971

Baguley IJ. 2008b. The excitatory:inhibitory ratio model (EIR model): An integrative explanation of acute autonomic overactivity syndromes. Med Hypotheses 70:26-35. 10.1016/j.mehy.2007.04.037

Baguley IJ, Heriseanu RE, Felmingham KL, and Cameron ID. 2006. Dysautonomia and heart rate variability following severe traumatic brain injury. 
Brain Inj 20:437-444. 10.1080/02699050600664715

Baguley IJ, Heriseanu RE, Gurka JA, Nordenbo A, and Cameron ID. 2007a. Gabapentin in the management of dysautonomia following severe traumatic brain injury: a case series. J Neurol Neurosurg Psychiatry 78:539-541. 10.1136/jnnp.2006.096388

Baguley IJ, Heriseanu RE, Nott MT, Chapman J, and Sandanam J. 2009a. Dysautonomia after severe traumatic brain injury: evidence of persisting overresponsiveness to afferent stimuli. Am J Phys Med Rehabil 88:615-622. 10.1097/PHM.0b013e3181aeab96

Baguley IJ, Nott MT, Slewa-Younan S, Heriseanu RE, and Perkes IE. 2009b. Diagnosing dysautonomia after acute traumatic brain injury: evidence for overresponsiveness to afferent stimuli. Arch Phys Med Rehabil 90:580-586. 10.1016/j.apmr.2008.10.020

Baguley IJ, Perkes IE, Fernandez-Ortega JF, Rabinstein AA, Dolce G, Hendricks HT, and Consensus Working G. 2014. Paroxysmal sympathetic hyperactivity after acquired brain injury: consensus on conceptual definition, nomenclature, and diagnostic criteria. J Neurotrauma 31:1515-1520. 10.1089/neu.2013.3301

Baguley IJ, Slewa-Younan S, Heriseanu RE, Nott MT, Mudaliar Y, and Nayyar V. 2007b. The incidence of dysautonomia and its relationship with autonomic arousal following traumatic brain injury. Brain Inj 21:1175-1181. $10.1080 / 02699050701687375$

Becker R, Benes L, Sure U, Hellwig D, and Bertalanffy H. 2000. Intrathecal baclofen alleviates autonomic dysfunction in severe brain injury. J Clin Neurosci 7:316-319. 10.1054/jocn.1999.0227

Bhigjee AI, Ames FR, and Rutherford GS. 1985. Adult aqueduct stenosis and diencephalic epilepsy. A case report. J Neurol Sci 71:77-89.

Blackman JA, Patrick PD, Buck ML, and Rust RS, Jr. 2004. Paroxysmal autonomic instability with dystonia after brain injury. Arch Neurol 61:321-328. 10.1001/archneur.61.3.321

Boeve BF, Wijdicks EF, Benarroch EE, and Schmidt KD. 1998. Paroxysmal 
sympathetic storms ("diencephalic seizures") after severe diffuse axonal head injury. Mayo Clin Proc 73:148-152. 10.1016/s0025-6196(11)63647-1

Bullard DE. 1987. Diencephalic seizures: responsiveness to bromocriptine and morphine. Ann Neurol 21:609-611. 10.1002/ana.410210617

Cai Y, Xu H, Yan J, Zhang L, and Lu Y. 2014. Molecular targets and mechanism of action of dexmedetomidine in treatment of ischemia/reperfusion injury. Mol Med Rep 9:1542-1550. 10.3892/mmr.2014.2034

Choi HA, Jeon SB, Samuel S, Allison T, and Lee K. 2013. Paroxysmal sympathetic hyperactivity after acute brain injury. Curr Neurol Neurosci Rep 13:370. 10.1007/s11910-013-0370-3

Cosar M, Eser O, Fidan H, Sahin O, Buyukbas S, Ela Y, Yagmurca M, and Ozen OA. 2009. The neuroprotective effect of dexmedetomidine in the hippocampus of rabbits after subarachnoid hemorrhage. Surg Neurol 71:54-59; discussion 59. 10.1016/j.surneu.2007.08.020

Cuny E, Richer E, and Castel JP. 2001. Dysautonomia syndrome in the acute recovery phase after traumatic brain injury: relief with intrathecal Baclofen therapy. Brain Inj 15:917-925. 10.1080/02699050110065277

Dahmani S, Rouelle D, Gressens P, and Mantz J. 2005. Effects of dexmedetomidine on hippocampal focal adhesion kinase tyrosine phosphorylation in physiologic and ischemic conditions. Anesthesiology 103:969-977.

Do D, Sheen VL, and Bromfield E. 2000. Treatment of paroxysmal sympathetic storm with labetalol. J Neurol Neurosurg Psychiatry 69:832-833.

Dolce G, Quintieri M, Leto E, Milano M, Pileggi A, Lagani V, and Pignolo L. 2008. Dysautonomia and Clinical Outcome in Vegetative State. J Neurotrauma. 10.1089/neu.2008.0536

Faul F, Erdfelder E, Lang AG, and Buchner A. 2007. G*Power 3: a flexible statistical power analysis program for the social, behavioral, and biomedical sciences. Behav Res Methods 39:175-191.

Fearnside MR, Cook RJ, McDougall P, and McNeil RJ. 1993. The Westmead Head Injury Project outcome in severe head injury. A comparative analysis of pre-hospital, 
clinical and CT variables. Br J Neurosurg 7:267-279.

Fernandez-Ortega JF, Prieto-Palomino MA, Garcia-Caballero M, Galeas-Lopez JL, Quesada-Garcia G, and Baguley IJ. 2012. Paroxysmal sympathetic hyperactivity after traumatic brain injury: clinical and prognostic implications. J Neurotrauma 29:13641370. 10.1089/neu.2011.2033

Fernandez-Ortega JF, Prieto-Palomino MA, Munoz-Lopez A, Lebron-Gallardo M, Cabrera-Ortiz H, and Quesada-Garcia G. 2006. Prognostic influence and computed tomography findings in dysautonomic crises after traumatic brain injury. J Trauma 61:1129-1133. 10.1097/01.ta.0000197634.83217.80

Goddeau RP, Jr., Silverman SB, and Sims JR. 2007. Dexmedetomidine for the treatment of paroxysmal autonomic instability with dystonia. Neurocrit Care 7:217220. 10.1007/s12028-007-0066-0

Greer DM, Funk SE, Reaven NL, Ouzounelli M, and Uman GC. 2008. Impact of fever on outcome in patients with stroke and neurologic injury: a comprehensive meta-analysis. Stroke 39:3029-3035. 10.1161/strokeaha.108.521583

Hendricks HT, Geurts AC, van Ginneken BC, Heeren AJ, and Vos PE. 2007. Brain injury severity and autonomic dysregulation accurately predict heterotopic ossification in patients with traumatic brain injury. Clin Rehabil 21:545-553. $10.1177 / 0269215507075260$

Hinson HE, and Sheth KN. 2012. Manifestations of the hyperadrenergic state after acute brain injury. Curr Opin Crit Care 18:139-145. 10.1097/MCC.0b013e3283513290

Jennett B, and Bond M. 1975. Assessment of outcome after severe brain damage. Lancet 1:480-484.

Kern J, Bodek D, Niazi OT, and Maher J. 2016. Refractory Case of Paroxysmal Autonomic Instability With Dystonia Syndrome Secondary to Hypoxia. Chest 149:e39-40. 10.1016/j.chest.2015.08.003

Ko SB, Kim CK, Lee SH, Bae HJ, and Yoon BW. 2010. Morphine-sensitive paroxysmal sympathetic storm in pontine intracerebral hemorrhage. Neurologist 16:384-385. 10.1097/NRL.0b013e3181b1cdff 
Lv LQ, Hou LJ, Yu MK, Qi XQ, Chen HR, Chen JX, Hu GH, Luo C, and Lu YC. 2010. Prognostic influence and magnetic resonance imaging findings in paroxysmal sympathetic hyperactivity after severe traumatic brain injury. J Neurotrauma 27:1945-1950. 10.1089/neu.2010.1391

Lv LQ, Hou LJ, Yu MK, Qi XQ, Chen HR, Chen JX, Hu GH, Luo C, and Lu YC. 2011. Risk factors related to dysautonomia after severe traumatic brain injury. J Trauma 71:538-542. 10.1097/TA.0b013e31820ebee1

Martin E, Ramsay G, Mantz J, and Sum-Ping ST. 2003. The role of the alpha2adrenoceptor agonist dexmedetomidine in postsurgical sedation in the intensive care unit. J Intensive Care Med 18:29-41. 10.1177/0885066602239122

Mehta NM, Bechard LJ, Leavitt K, and Duggan C. 2008. Severe weight loss and hypermetabolic paroxysmal dysautonomia following hypoxic ischemic brain injury: the role of indirect calorimetry in the intensive care unit. JPEN J Parenter Enteral Nutr 32:281-284. 10.1177/0148607108316196

Nelson LE, Lu J, Guo T, Saper CB, Franks NP, and Maze M. 2003. The alpha2adrenoceptor agonist dexmedetomidine converges on an endogenous sleeppromoting pathway to exert its sedative effects. Anesthesiology 98:428-436.

Panzer O, Moitra V, and Sladen RN. 2011. Pharmacology of sedative-analgesic agents: dexmedetomidine, remifentanil, ketamine, volatile anesthetics, and the role of peripheral $\mathrm{Mu}$ antagonists. Anesthesiol Clin 29:587-605, vii. 10.1016/j.anclin.2011.09.002

Penfield W. 1929. DIencephalic autonomic epilepsy. Archives of Neurology \& Psychiatry 22:358-374. 10.1001/archneurpsyc.1929.02220020174010

Perkes I, Baguley IJ, Nott MT, and Menon DK. 2010. A review of paroxysmal sympathetic hyperactivity after acquired brain injury. Ann Neurol 68:126-135. 10.1002/ana.22066

Perkes IE, Menon DK, Nott MT, and Baguley IJ. 2011. Paroxysmal sympathetic hyperactivity after acquired brain injury: a review of diagnostic criteria. Brain Inj 25:925-932. 10.3109/02699052.2011.589797

Pranzatelli MR, Pavlakis SG, Gould RJ, and De Vivo DC. 1991. Hypothalamic- 
midbrain dysregulation syndrome: hypertension, hyperthermia, hyperventilation, and decerebration. J Child Neurol 6:115-122.

Rabinstein AA. 2007. Paroxysmal sympathetic hyperactivity in the neurological intensive care unit. Neurol Res 29:680-682. 10.1179/016164107x240071

Rabinstein AA, and Benarroch EE. 2008. Treatment of paroxysmal sympathetic hyperactivity. Curr Treat Options Neurol 10:151-157.

Russo RN, and O'Flaherty S. 2000. Bromocriptine for the management of autonomic dysfunction after severe traumatic brain injury. J Paediatr Child Health 36:283-285.

Sneed RC. 1995. Hyperpyrexia associated with sustained muscle contractions: an alternative diagnosis to central fever. Arch Phys Med Rehabil 76:101-103.

Thorley RR, Wertsch JJ, and Klingbeil GE. 2001. Acute hypothalamic instability in traumatic brain injury: a case report. Arch Phys Med Rehabil 82:246-249. 10.1053/apmr.2001.18698

Tracey KJ. 2007. Physiology and immunology of the cholinergic antiinflammatory pathway. J Clin Invest 117:289-296. 10.1172/jci30555

Venn RM, Hell J, and Grounds RM. 2000. Respiratory effects of dexmedetomidine in the surgical patient requiring intensive care. Crit Care 4:302308. $10.1186 /$ cc712 
Figure 1

Ratio of patients meeting different criteria regarding likelihood of PSH diagnosis

The ratio of patients who meet the criteria of "probable", "possible", and "unlikely" respectively, in the dexmedetomidine group and in the control group. * indicates significance $(p=0.028)$.

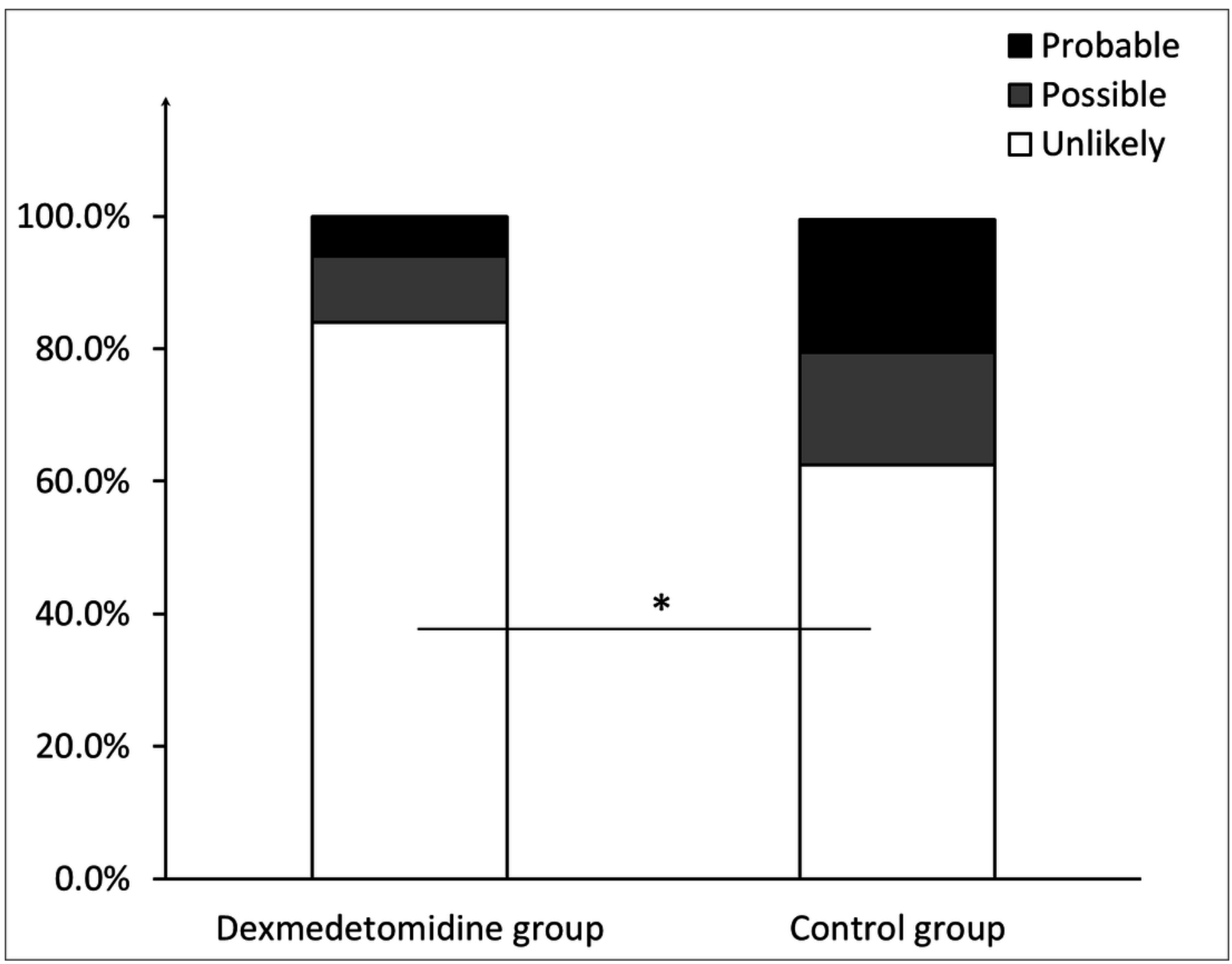


Figure 2

Scores of patients meeting different criteria regarding likelihood of PSH diagnosis

The comparison of the PSH scores of the patients who meet the criteria of "probable", "possible", and "unlikely", respectively, between the dexmedetomidine group and the control group. $*$ indicates significance $(p=0.045)$.

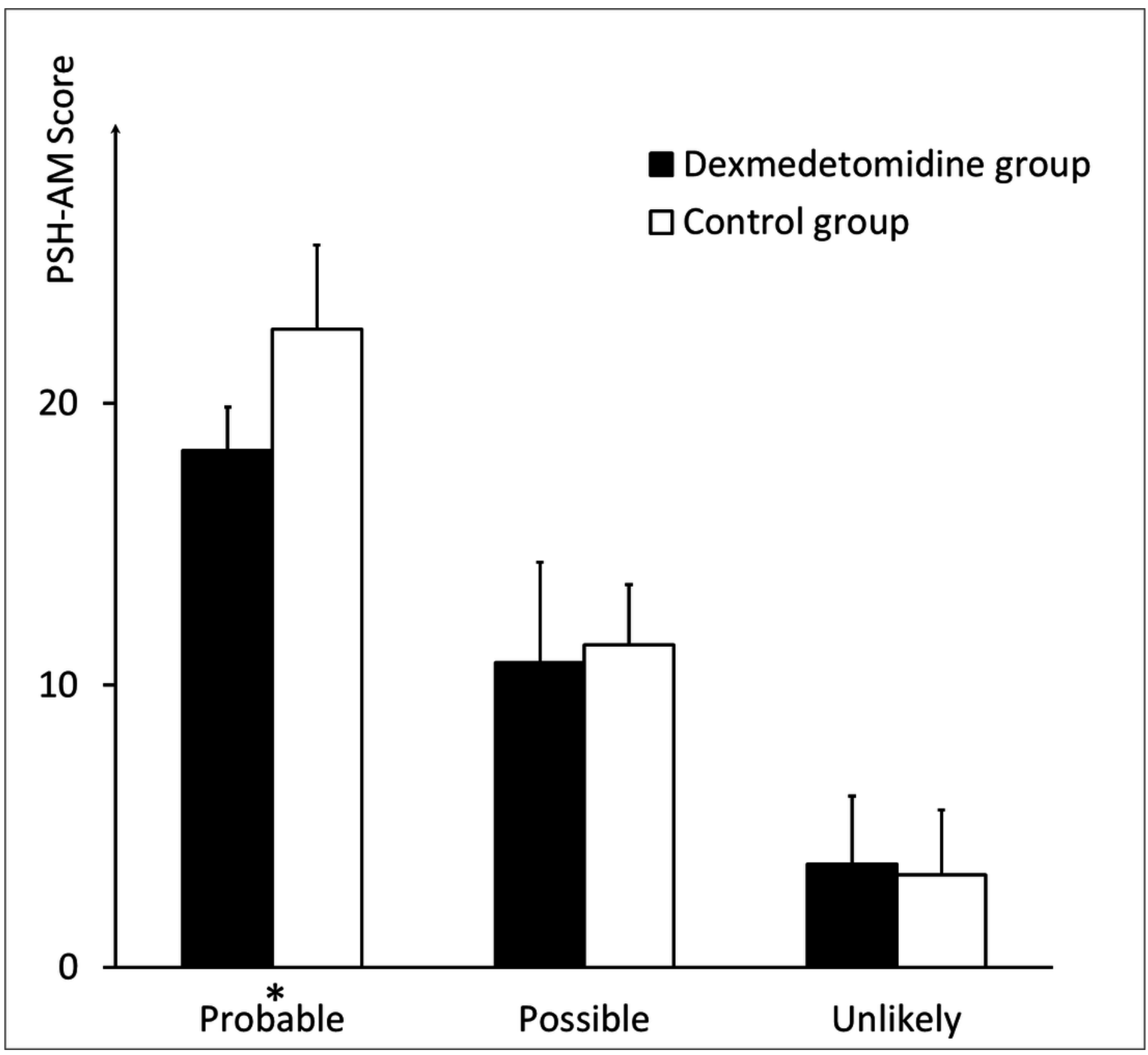


Figure 3

NICU and hospital stay of the two groups

The comparison of the duration of the NICU and hospital stays between the dexmedetomidine group and the control group.

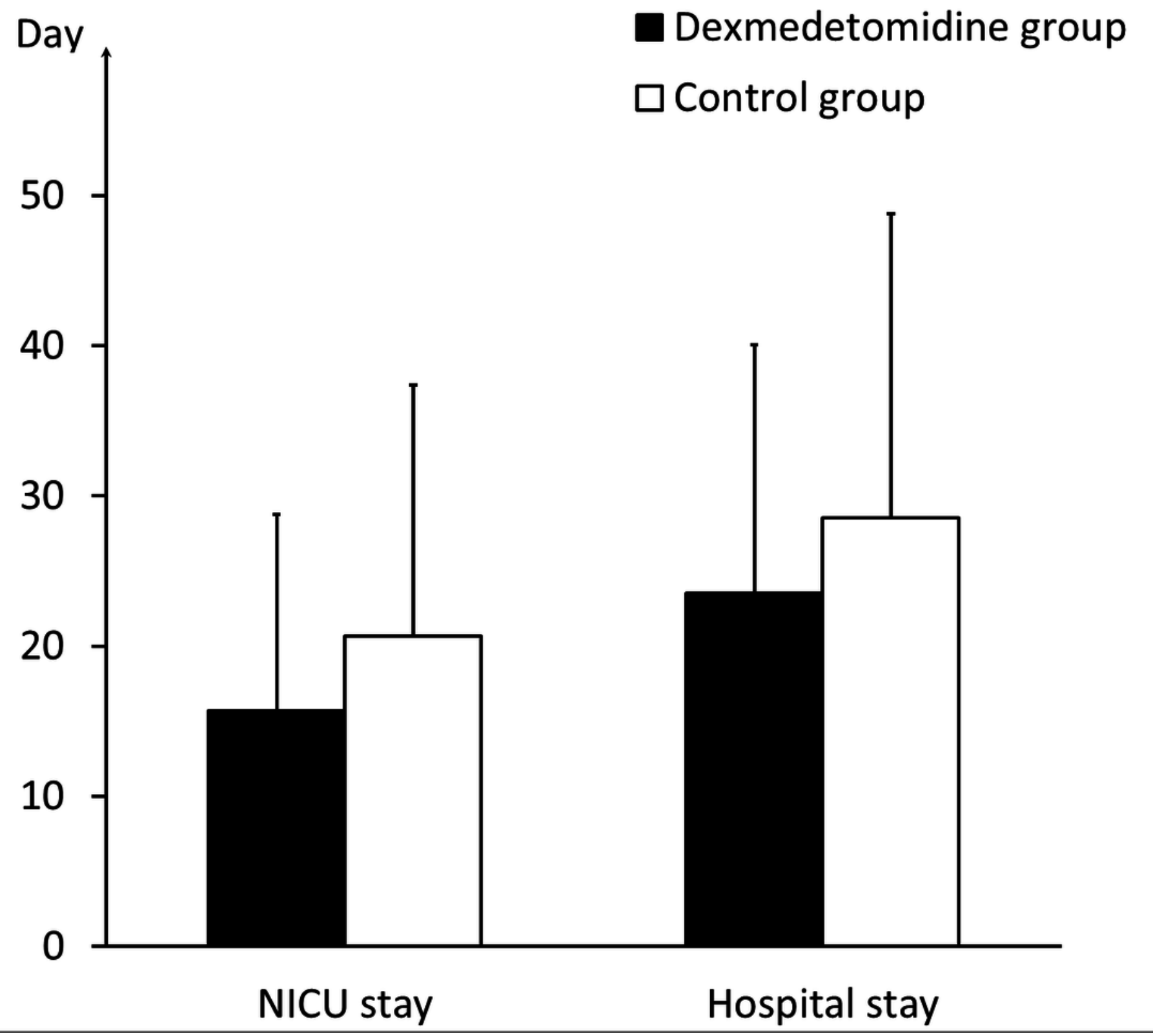


Figure 4

Outcome of the two groups

The preoperative GCS, GOS at discharge, and GOS after three months in the dexmedetomidine group and the control group.

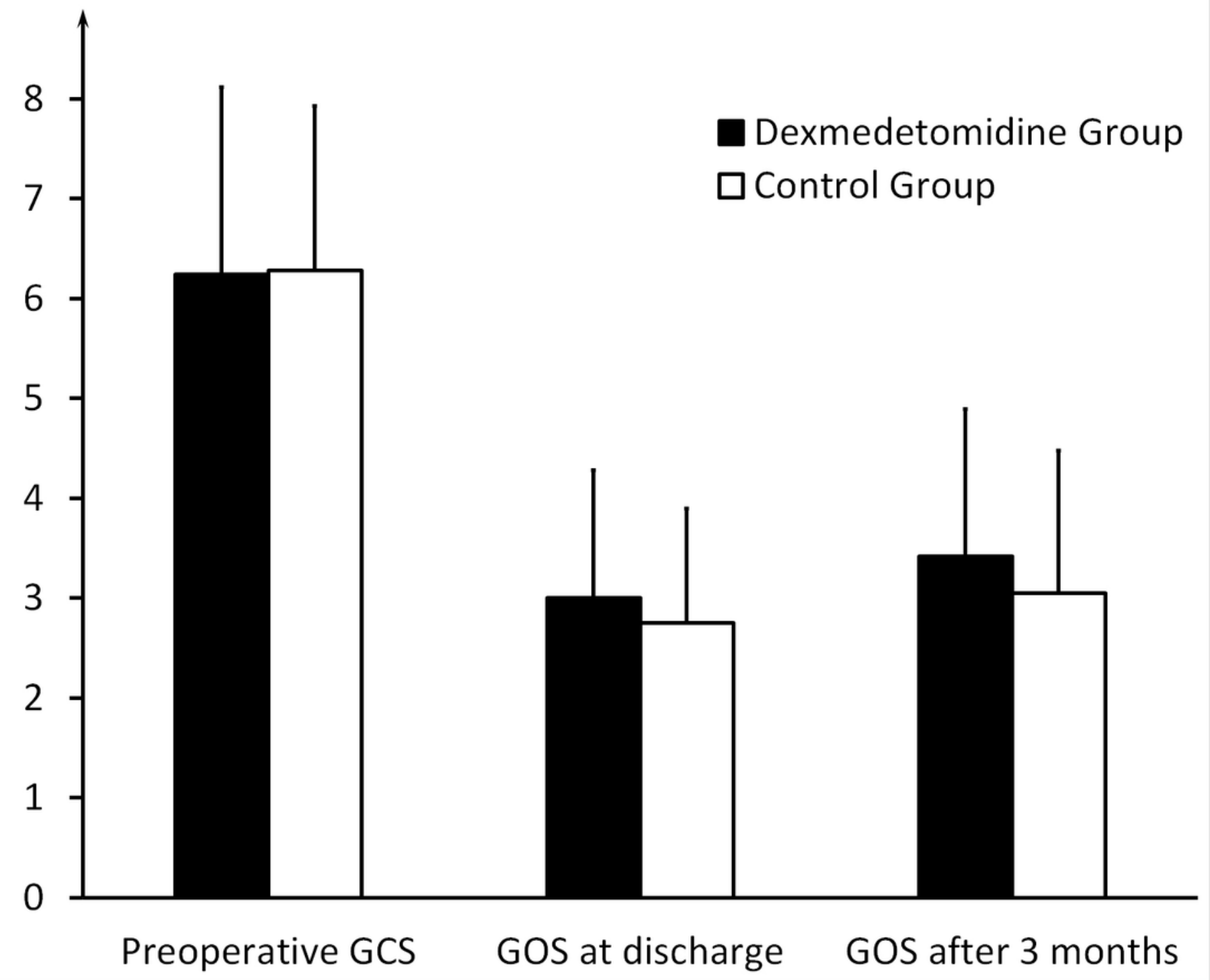




\section{Table $\mathbf{1}$ (on next page)}

Baseline demographic, clinical characteristics, PSH-AM score and outcome of the study population

Patients' age, gender, pre-operative GCS, and diagnosis, time from injury to surgery, type of surgery, day of sedation, the ratio and the PSH-AM score of the patients who met the criteria of "probable", "probable" and "unlikely", the NICU and Hospital stays, and GOS at discharge and after three months. 


\begin{tabular}{|c|c|c|c|c|}
\hline Variables & Total & $\begin{array}{c}\text { Dexmedetomidine } \\
\text { group }\end{array}$ & $\begin{array}{l}\text { Control } \\
\text { group }\end{array}$ & $p$ vaule \\
\hline \multicolumn{5}{|l|}{ Demographic } \\
\hline No. of patients & 90 & 50 & 40 & - \\
\hline Male gender & $62(68.9 \%)$ & $37(74 \%)$ & $25(62.5 \%)$ & 0.242 \\
\hline Age, Mean $\pm S D, y$ & $46.76 \pm 15.41$ & $47.50 \pm 15.12$ & $45.83 \pm 15.92$ & 0.611 \\
\hline Preoperative GCS, Mean \pm SD & $6.26 \pm 1.77$ & $6.24 \pm 1.88$ & $6.28 \pm 1.65$ & 0.926 \\
\hline \multicolumn{5}{|l|}{ Diagnosis } \\
\hline Cerebral contusion without hemorrhage & $23(25.6 \%)$ & $14(28 \%)$ & $9(22.5 \%)$ & 0.552 \\
\hline Hemorrhagic cerebral contusion & $54(60 \%)$ & $30(60 \%)$ & $24(60 \%)$ & 1 \\
\hline Acute subdural hematoma & $72(80 \%)$ & $38(76 \%)$ & $34(85 \%)$ & 0.289 \\
\hline Acute epidural hematoma & $35(38.9 \%)$ & $19(38 \%)$ & $16(40 \%)$ & 0.847 \\
\hline Skull fracture & $65(72.2 \%)$ & $36(72 \%)$ & $29(72 \%)$ & 0.958 \\
\hline Subarachnoid hemorrhage & $78(86.7 \%)$ & $43(86 \%)$ & $35(87 \%)$ & 0.835 \\
\hline Cerebral hernia & $30(33.3 \%)$ & $14(28 \%)$ & $16(40 \%)$ & 0.23 \\
\hline Time from injury to surgery & $5.21 \pm 1.75$ & $5.03 \pm 1.76$ & $5.43 \pm 1.71$ & 0.89 \\
\hline \multicolumn{5}{|l|}{ Type of surgery } \\
\hline Decompressive craniectomy & $87(96.7 \%)$ & $48(96 \%)$ & $39(97.5 \%)$ & 1 \\
\hline Epidural hematoma clearing & $35(38.9 \%)$ & $19(38 \%)$ & $16(40 \%)$ & 0.847 \\
\hline Subdural hematoma clearing & $72(80 \%)$ & $38(76 \%)$ & $34(85 \%)$ & 0.289 \\
\hline Hemorrhagic contusion clearing & $47(52.2 \%)$ & $25(50 \%)$ & $22(55 \%)$ & 0.637 \\
\hline Lateral ventriculopuncture drainage & $90(100 \%)$ & $50(100 \%)$ & $40(100 \%)$ & 1 \\
\hline Day of sedation & $5.73 \pm 2.88$ & $5.46 \pm 2.82$ & $6.08 \pm 2.95$ & 0.317 \\
\hline PSH diagnostic likelihood & $6.73 \pm 6.58$ & $5.26 \pm 4.66$ & $8.58 \pm 8.09$ & 0.017 \\
\hline \multicolumn{5}{|l|}{ Probable $(\geqslant 17)$} \\
\hline No. & $11(12.2 \%)$ & $3(6 \%)$ & $8(20.0 \%)$ & 0.056 \\
\hline Score & $21.45 \pm 3.27$ & $18.33 \pm 1.53$ & $22.63 \pm 2.97$ & 0.045 \\
\hline \multicolumn{5}{|l|}{ Possible (8-16) } \\
\hline No. & $12(13.3 \%)$ & $5(10 \%)$ & $7(17.5 \%)$ & 0.358 \\
\hline Score & $11.17 \pm 2.69$ & $10.80 \pm 3.56$ & $11.43 \pm 2.15$ & 0.710 \\
\hline \multicolumn{5}{|l|}{ Unlikely $(<8)$} \\
\hline No. & $67(74.4 \%)$ & $42(84 \%)$ & $25(62.5 \%)$ & 0.028 \\
\hline Score & $3.52 \pm 2.35$ & $3.67 \pm 2.40$ & $3.28 \pm 2.30$ & 0.519 \\
\hline NICU stay, Mean $\pm S D, d$ & $17.90 \pm 14.93$ & $15.70 \pm 13.07$ & $20.65 \pm 16.74$ & 0.119 \\
\hline Hospital stay, Mean $\pm S D, d$ & $25.87 \pm 18.40$ & $23.50 \pm 16.58$ & $28.53 \pm 20.28$ & 0.174 \\
\hline GOS at discharge & $2.89 \pm 1.22$ & $3.00 \pm 1.28$ & $2.75 \pm 1.15$ & 0.338 \\
\hline GOS after 3 months & $3.25 \pm 1.46$ & $3.42 \pm 1.47$ & $3.05 \pm 1.43$ & 0.234 \\
\hline
\end{tabular}

Note: $\mathrm{P}$ Values for differences between two treatment groups by Student $\mathrm{t}$ test or Fisher's exact test. 